Gut and Liver, Vol. 13, No. 1, January 2019, pp. 62-66

\title{
Distinction between Chronic Enteropathy Associated with the SLC02A1 Gene and Crohn's Disease
}

Shunichi Yanai ${ }^{1}$, Satoko Yamaguchi ${ }^{1}$, Shotaro Nakamura ${ }^{1}$, Keisuke Kawasaki ${ }^{1}$, Yosuke Toya ${ }^{1}$, Noriyuki Yamada ${ }^{2}$, Makoto Eizuka $^{2}$, Noriyuki Uesugi ${ }^{2}$, Junji Umeno ${ }^{3}$, Motohiro Esaki ${ }^{4}$, Eiko Okimoto ${ }^{5}$, Shunji Ishihara ${ }^{5}$, Tamotsu Sugai ${ }^{2}$, and Takayuki Matsumoto $^{1}$

${ }^{1}$ Division of Gastroenterology, Department of Internal Medicine and ${ }^{2}$ Division of Molecular Diagnostic Pathology, Department of Pathology, Iwate Medical University, Morioka, ${ }^{3}$ Department of Medicine and Clinical Science, Graduate School of Medical Sciences, Kyushu University, Fukuoka, ${ }^{4}$ Department of Endoscopic Diagnostic and Therapeutics, Saga University, Saga, and ${ }^{5}$ Department of Gastroenterology and Hepatology, Shimane University School of Medicine, Izumo, Japan

Background/Aims: We recently identified recessive mutations in the solute carrier organic anion transporter family member $2 \mathrm{~A} 1$ gene (SLCO2A1) as causative variants of chronic nonspecific multiple ulcers of the small intestine (chronic enteropathy associated with SLCO2A1, CEAS). The aim of this study was to investigate the gastroduodenal expression of the SLCO2A1 protein in patients with CEAS and Crohn's disease (CD). Methods: Immunohistochemical staining for SLCO2A1 was performed with a polyclonal antibody, HPA013742, on gastroduodenal tissues obtained by endoscopic biopsy from four patients with CEAS and 29 patients with CD. Results: The expression of SLCO2A1 was observed in one of four patients (25\%) with CEAS and in all 29 patients $(100 \%)$ with CD $(p<0.001)$. The three patients with CEAS without SLCO2A1 expression had a homozygous splice-site mutation in SLCO2A1, c.1461+1G>C (exon 7) or c.940+1G>A (exon 10). The remaining one CEAS patient with positive expression of SLCO2A1 had compound heterozygous c.664G $>$ A and c.1807C>T mutations. Conclusions: Immunohistochemical staining for SLCO2A1 in gastroduodenal tissues obtained by endoscopic biopsy is considered useful for the distinction of CEAS from CD. (Gut Liver 2019;13:62-66)

Key Words: SLCO2A1; Chronic enteropathy associated with SLCO2A1 gene; Crohn disease; Immunohistochemistry

\section{INTRODUCTION}

Chronic nonspecific multiple ulcers of the small intestine (CNSU) is a rare autosomal recessive inherited disease character- ized by chronic loss of blood and protein through persistent, intractable nonspecific small intestinal ulcers. ${ }^{1-5}$ The ulcers in CNSU occur predominantly in the ileum, while the terminal ileum is usually intact. The ulcers are multiple (usually $>20$ ) and each lesion manifests a shallow and flat ulcer bed surrounded by a discrete margin. The configuration of each ulcer is usually linear or a tall triangle, and the ulcer is aligned circularly or obliquely. The ulcers occasionally fuse, thus showing a geographic configuration..$^{1-5}$

In 2015, we identified the solute carrier organic anion transporter family member $2 \mathrm{~A} 1$ gene (SLCO2A1) as the causative gene for CNSU, and we suggested a more appropriate nomenclature of "chronic enteropathy associated with SLCO2A1 (CEAS)" for the disease. ${ }^{6,7}$ In 2016, Uchida et al. ${ }^{8}$ conducted a Japanese nationwide clinical and genetic survey of pediatric patients with CEAS, and identified four children with the disease. On the basis of their results, Uchida et al. ${ }^{8}$ proposed that CEAS should be seriously considered as a differential diagnosis for pediatric patients suspected of having inflammatory bowel disease, especially for those of Crohn's disease (CD). However, it is often difficult to distinguish CEAS from $\mathrm{CD}$, since both diseases affect the small bowel with intractable chronic ulcers. ${ }^{9,10}$

Recently, we evaluated SLCO2A1 protein expression in the intestinal tissues of patients with CEAS. ${ }^{11}$ In the present study, we evaluated the immunohistochemical expression of the SLC02A1 protein in gastroduodenal tissues obtained by endoscopic biopsy from patients with CEAS and CD to examine whether biopsy specimens can be used for distinguishing these diseases from each other.

Correspondence to: Shunichi Yanai

Division of Gastroenterology, Department of Internal Medicine, Iwate Medical University, Uchimaru 19-1, Morioka 020-8505, Japan

Tel: +81-19-651-5111, Fax: +81-19-652-6664, E-mail: syanai@iwate-med.ac.jp

Received on June 6, 2018. Revised on July 18, 2018. Accepted on July 30, 2018. Published online January 3, 2019

pISSN 1976-2283 eISSN 2005-1212 https://doi.org/10.5009/gnl18261

@ This is an Open Access article distributed under the terms of the Creative Commons Attribution Non-Commercial License (http://creativecommons.org/licenses/by-nc/4.0) which permits unrestricted non-commercial use, distribution, and reproduction in any medium, provided the original work is properly cited. 


\section{MATERIALS AND METHODS}

\section{Patients}

We reviewed the clinical files of patients diagnosed with inflammatory bowel diseases at our institutions between 2001 and 2014. There were 29 patients with CD and four patients with CEAS. Characteristics in three of the four patients with CEAS had been described in the previous study, ${ }^{11}$ while all the 29 patients with $\mathrm{CD}$ were completely different from those in the previous study. All 33 patients had undergone esophagogastroduodenoscopy with multiple endoscopic biopsies from the normalappearing gastroduodenal mucosa. In 23 patients with CD and three patients with CEAS, biopsy specimens were obtained from both the gastric and duodenal mucosa. In other four patients with $\mathrm{CD}$ and the remaining one patient with CEAS, the specimens were taken only from the duodenum. In the remaining two patients with $\mathrm{CD}$, that was taken only from the stomach. The mean number of the biopsy specimens was 3.6 in CD cases and 3.5 in CEAS cases (Table 1). The diagnosis of CD was based on the established Japanese criteria. ${ }^{12}$ All study participants provided written informed consent for this analysis. The study protocol was approved by the ethics committee at Iwate Medical University Hospital (H28-59; August 4, 2016) and Kyushu Uni-

Table 1. Clinicopathologic Features of Study Patients

\begin{tabular}{|c|c|c|}
\hline Variable & $C D(n=29)$ & CEAS $(n=4)$ \\
\hline Age at diagnosis, yr & $23(9-52)$ & $28(22-68)$ \\
\hline Age at biopsy, yr & $31(16-56)$ & $63(53-75)$ \\
\hline \multicolumn{3}{|l|}{ Sex } \\
\hline Male & 19 & 1 \\
\hline Female & 10 & 3 \\
\hline \multicolumn{3}{|l|}{ Involved intestinal portion } \\
\hline Ileum only & 2 & 4 \\
\hline Colorectal involvement & 27 & 0 \\
\hline \multicolumn{3}{|l|}{ Laboratory data } \\
\hline Albumin, g/dL & $3.1 \pm 0.8$ & $2.9 \pm 0.6$ \\
\hline $\mathrm{CRP}, \mathrm{mg} / \mathrm{dL}$ & $2.3 \pm 2.4$ & $0.8 \pm 1.1$ \\
\hline Hemoglobin, g/dL & $11.6 \pm 2.4$ & $10.2 \pm 1.7$ \\
\hline \multicolumn{3}{|l|}{ Medication } \\
\hline 5-ASAs & 25 & 1 \\
\hline Corticosteroids & 11 & 3 \\
\hline Immunomodulators (AZA/6-MP) & 18 & 1 \\
\hline Biologics (IFX/ADA) & 25 & 0 \\
\hline No. of specimens/case & $3.6 \pm 0.9$ & $3.5 \pm 1.9$ \\
\hline Expression of SLCO2A1 & $29(100)$ & $1(25)$ \\
\hline
\end{tabular}

Data are presented as median (range), mean $\pm \mathrm{SD}$, or number (\%). $\mathrm{CD}$, Crohn's disease; CEAS, chronic enteropathy associated with SLCO2A1; CRP, C-reactive protein; 5-ASAs, aminosalicylates; AZA, azathioprine; 6-MP, 6-mercaptopurine; IFX, infliximab; ADA, adalimumab. versity (439-06; January 26, 2015). The study was conducted in accordance with the Helsinki Declaration.

\section{Genetic analysis of the SLCO2A1 gene}

In all four cases of CEAS, mutations in the SLCO2A1 gene were analyzed, as described previously. ${ }^{6}$ Briefly, DNA was extracted from peripheral blood using standard methods, and Sanger sequencing of exons 4, 5, 7, 10 and 13 of the SLCO2A1 gene was performed to identify the mutation sites. ${ }^{6}$ In the 29 cases of $\mathrm{CD}$, however, mutations in the SLCO2A1 gene were not analyzed.

\section{Immunohistochemical analysis of SLCO2A1 protein}

In all 33 cases, immunohistochemical staining was performed on 4- $\mu \mathrm{m}$ thick sections cut from each of the formalin-fixed and paraffin-embedded tissue blocks with a polyclonal antibody for SLCO2A1 (HPA013742; Sigma-Aldrich, St. Louis, MO, USA) and a CD31 monoclonal antibody (Santa Cruz Biotechnology, Santa Cruz, CA, USA) using the Dako Envision system (Dako, Glostrup, Denmark) according to the manufacturer's instructions.

The immune expression of SLCO2A1 in vascular endothelial cells, as confirmed by CD31 immunostaining, in the respective lesions was determined to be either positive or negative by two independent observers (S.Yanai and N.U.).

\section{Statistical analysis}

Differences between groups were evaluated with a Fisher exact test, chi-square test or a Mann-Whitney U-test as appropriate. All p-values $<0.05$ were regarded as statistically significant.

\section{RESULTS}

The clinicopathologic features of the study patients are summarized in Table 1. While the median age at diagnosis did not differ between patients with CD (23 years) and patients with CEAS (28 years), the median age at biopsy was significantly younger in $\mathrm{CD}$ patients ( 31 years) than in CEAS patients (63 years, $\mathrm{p}<0.001$ ). Patients with $\mathrm{CD}$ were predominantly male, while CEAS patients were predominantly female. However, the difference was insignificant. Colorectal involvement was confirmed in 27 patients with CD (93\%), but in none of the patients with CEAS $(\mathrm{p}<0.001)$. Laboratory data, including hemoglobin, albumin and C-reactive protein (CRP) levels did not differ significantly between CD and CEAS, though the mean CRP level was higher in CD $(2.3 \mathrm{mg} / \mathrm{dL})$ than in CEAS $(0.8 \mathrm{mg} / \mathrm{dL})$. Treatment with biologics (infliximab or adalimumab) was carried out in 25 of 29 patients (86\%) with CD, while none of the four patients with CEAS $(\mathrm{p}=0.001)$ were undergoing treatment with biologics.

SLCO2A1 expression in the gastroduodenal tissues was confirmed in all 29 patients with CD (100\%) (Fig. 1). In contrast, SLCO2A1 expression was found only in one of the four patients 

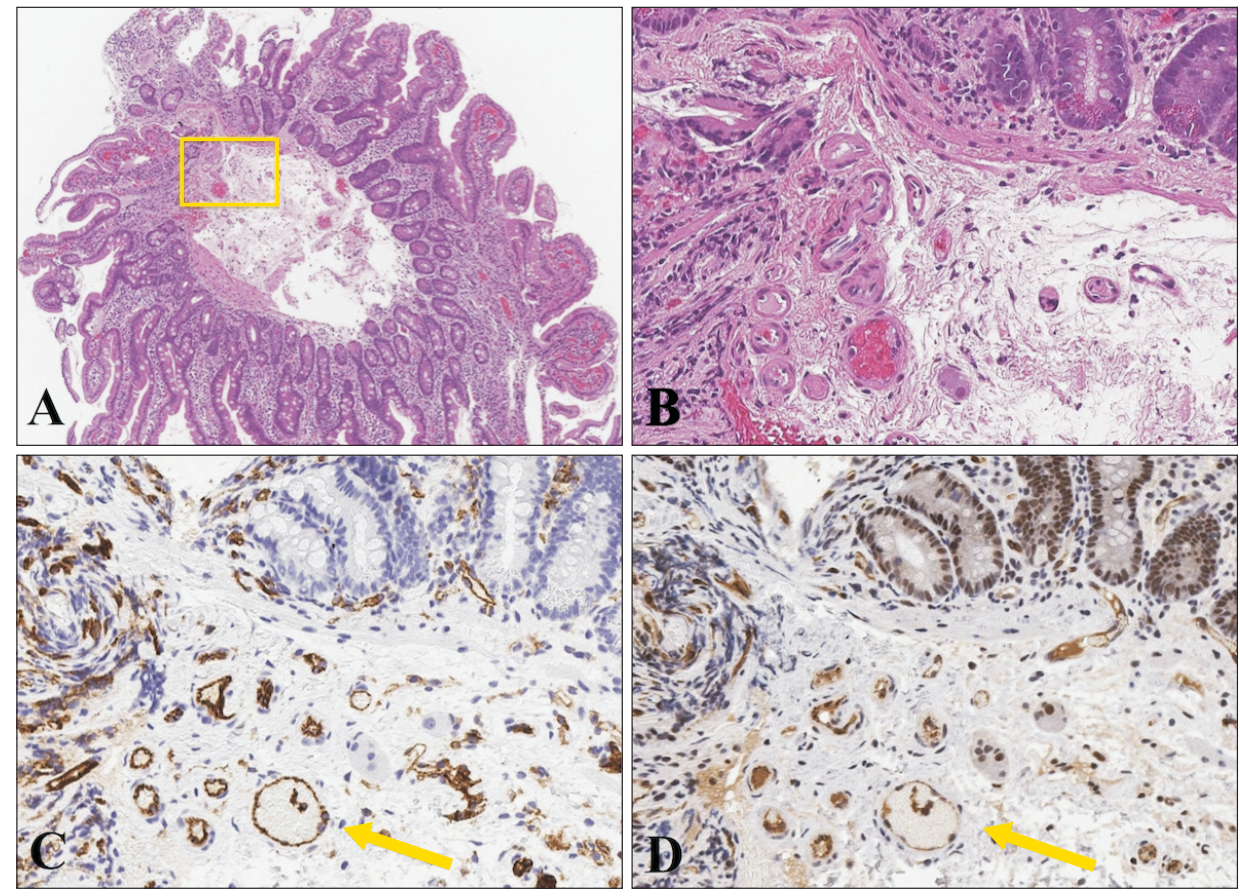

Fig. 1. Photomicrographs of the duodenal tissue obtained by endoscopic biopsy from a patient with Crohn's disease. (A) Low-power view showing the duodenal mucosa and submucosa with a mild chronic inflammatory infiltrate $(\mathrm{H} \& \mathrm{E}, \times 40)$. (B) Mid-power view showing several capillary vessels in the deep mucosa and superficial submucosa (H\&E, $\times 200$ ). (C) CD31 immunostaining highlights the vascular endothelial cells of the capillary vessels (arrow) in the mucosa and submucosa (×200). (D) SLCO2A1 immunostaining. Expression of the SLCO2A1 protein can be observed in the vascular endothelial cells (arrow) in the duodenal mucosa and submucosa (×200).
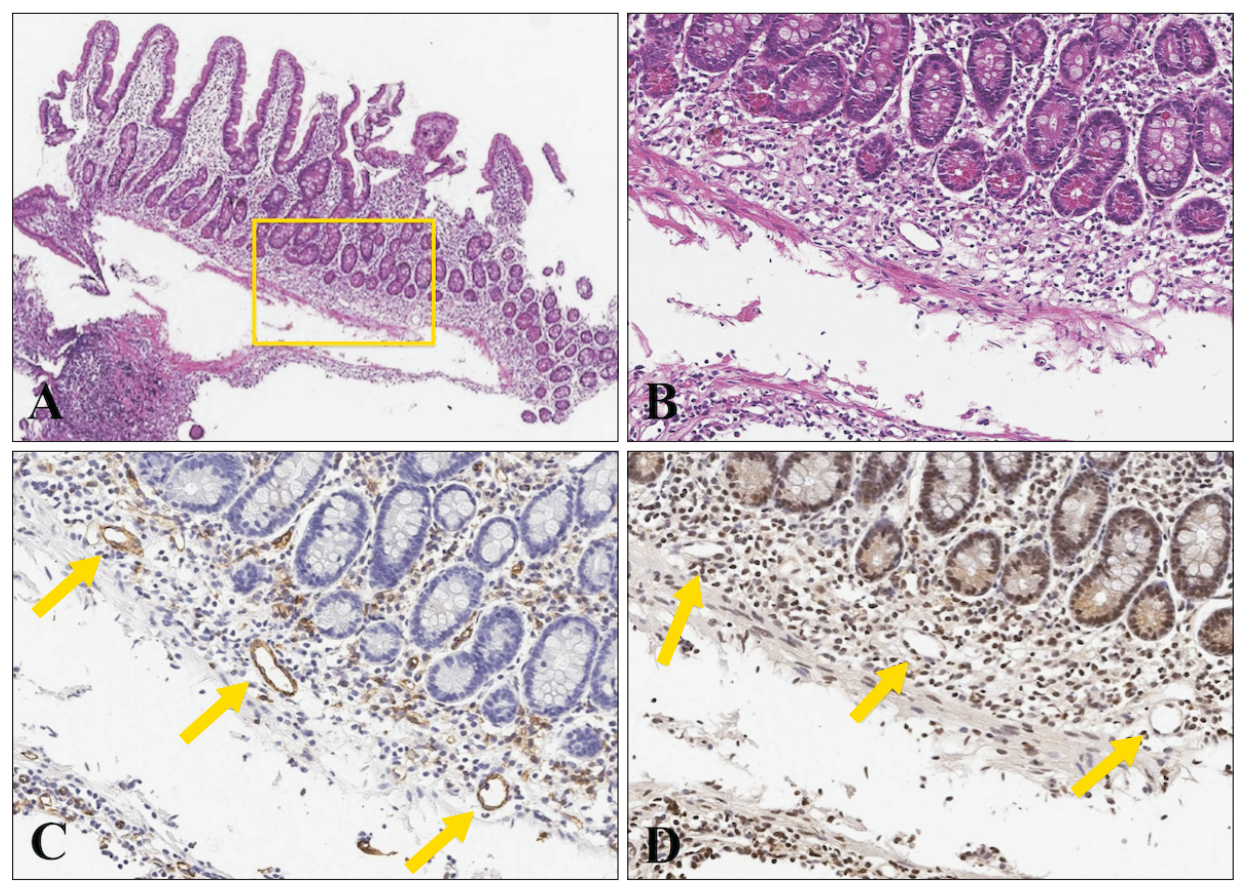

Fig. 2. Photomicrographs of the duodenal tissue obtained by endoscopic biopsy from a patient with chronic enteropathy associated with SLC02A1 (CEAS). (A) Low-power view showing the duodenal mucosa with a mild chronic inflammatory infiltrate (H\&E, ×40). (B) Mid-power view showing several capillary vessels in the deep portion of the mucosa, (H\&E, ×200). (C) CD31 immunostaining highlights the vascular endothelial cells of the capillary vessels in the mucosa (arrows) $(\times 200)$. (D) SLCO2A1 immunostaining. No SLCO2A1 protein expression can be observed in the vascular endothelial cells (arrows) in the duodenal mucosa $(\times 200)$.

with CEAS (25\%, p<0.001) (Table 1, Fig. 2). Both the gastric and duodenal tissues were examined in 23 of 29 patients with CD and in three of four patients with CEAS. Between gastric and duodenal tissues, SLCO2A1 expression pattern did not differ in all the 23 patients with $\mathrm{CD}$ and all the three patients with CEAS. In all the 23 patients with CD, SLCO2A1 was positive both in the gastric and duodenal tissues. In all the three patients with CEAS without SLCO2A1 expression, it was negative both in the gastric and duodenal tissues. As shown in Table 2, three of four patients with CEAS without SLCO2A1 expression (cases 1, 2, 4) had a homozygous splice-site mutation in the SLCO2A1 gene, c. $1461+1 \mathrm{G}>\mathrm{C}$ (exon 7) or c.940+1G>A (exon 10). The remaining CEAS patient (case 3) with positive expression of SLCO2A1 had compound heterozygous mutations of c.664G $>$ A and c. $1807 \mathrm{C}>\mathrm{T}$.

\section{DISCUSSION}

Development and widespread use of enteroscopy, such as bal- 
Table 2. SLCO2A1 Mutations and SLCO2A1 Expression in the 4 Patients with CEAS

\begin{tabular}{|c|c|c|c|c|c|c|c|c|}
\hline Case & $\begin{array}{c}\text { Age at biopsy, } \\
\text { yr }\end{array}$ & Sex & Exon & Pattern & Nucleotide change & $\begin{array}{l}\text { Mutant allele } \\
\text { frequency }^{6}\end{array}$ & $\begin{array}{l}\text { Amino acid } \\
\text { change }\end{array}$ & $\begin{array}{c}\text { SLC02A1 } \\
\text { protein }\end{array}$ \\
\hline 1 & 57 & Female & 10 & Homozygous & c. $1461+1 \mathrm{G}>\mathrm{C}$ c $.1461+1 \mathrm{G}>\mathrm{C}$ & $2 / 32$ & Splice & Negative \\
\hline 2 & 75 & Female & 7 & Homozygous & c. $940+1 \mathrm{G}>$ A c. $940+1 \mathrm{G}>$ A & $19 / 32$ & Splice & Negative \\
\hline \multirow[t]{2}{*}{3} & 53 & Female & 5 & Compound & c. $664 \mathrm{G}>\mathrm{A}$ & $4 / 32$ & Deleterious & Positive \\
\hline & & & 13 & heterozygous & c. $1807 \mathrm{C}>\mathrm{T}$ & $4 / 32$ & No & \\
\hline 4 & 69 & Male & 7 & Homozygous & c. $940+1 \mathrm{G}>$ A c $.940+1 \mathrm{G}>\mathrm{A}$ & $19 / 32$ & Splice & Negative \\
\hline
\end{tabular}

CEAS, chronic enteropathy associated with SLCO2A1.

loon endoscopy ${ }^{13}$ and capsule endoscopy, ${ }^{14}$ have enabled gastroenterologists to observe mucosal lesions of the whole small bowel. Therefore, the opportunities for diagnosing small bowel diseases have increased. However, the practical and accurate diagnosis of various ulcerative disorders of the small bowel, such as CD, intestinal Behçet's disease, CEAS, cryptogenic multifocal ulcerous stenosing enteritis ${ }^{15,16}$ and nonsteroidal anti-inflammatory drug induced enteropathy is a matter of debate, because those diseases are often difficult to distinguish from each other by endoscopic findings alone.

CEAS, previously referred to as CNSU, is a rare autosomal recessive inherited disease characterized by chronic blood and protein loss through persistent, intractable and histologically nonspecific small bowel ulcers. The SLCO2A1 gene, which encodes a prostaglandin $(\mathrm{PG})$ transporter, has been identified as a causative gene not only for CEAS, but also for a subtype of primary hypertrophic osteoarthropathy (PHO)/pachydermoperiostosis (PDP). ${ }^{17-21}$ Dysfunction of the SLCO2A1 protein would cause the failure of prostaglandin E2 (PGE2) re-uptake, resulting in high PGE2 in serum and urine.

Compton et al. ${ }^{9}$ and Shim and Suh ${ }^{10}$ previously reported family pedigrees of patients with $\mathrm{CD}$ combined with $\mathrm{PHO}$. On the basis of the above-mentioned identification of the SLCO2A1 gene, it can be speculated that the families had actually been suffering from CEAS rather than CD. In our previous study, ${ }^{6}$ two of 603 patients with a clinical diagnosis of $C D$ were found to have compound heterozygous mutations in SLCO2A1, and accordingly, we revised the diagnosis for those two patients as CEAS. It thus seems possible that cases of CEAS are clinically misinterpreted as CD, probably because of apparently indistinguishable clinical manifestations, such as small intestinal ulcers, anemia and hypoproteinemia. Genetic analysis is therefore mandatory for distinguishing CEAS from CD.

It has been confirmed that the SLCO2A1 protein is expressed on the cellular membrane of vascular endothelial cells within the small intestinal mucosa and submucosa in healthy subjects. ${ }^{6}$ In our previous study, loss of SLCO2A1 protein expression in the resected intestinal tissues was confirmed in two of three patients with CEAS, while the expression of the protein was positive in all 22 patients with $\mathrm{CD}$ or intestinal Behçet's disease. ${ }^{11}$ In the present study, we further confirmed similar findings in the gastroduodenal mucosal tissues obtained by endoscopic biopsy. As a result, loss of SLCO2A1 protein expression was evident in three of four patients with CEAS (75\%), but in none of the 29 patients with CD. It thus seems possible that in patients with loss of SLCO2A1 protein expression in biopsy specimens, genetic analysis for the SLCO2A1 gene may not be necessary for the clinical diagnosis of CEAS.

It should be noted that one of the cases of CEAS (case 3) was positive for SLCO2A1 protein expression, even though the case had compound heterozygous mutations of the SLCO2A1 gene. While the full and complete SLCO2A1 protein is composed of 643 amino acids, the antibody we used for the present study (HPA013742) detects only the 83 amino acids in the fifth extracellular domain encoded by exons 9-11 (431-513) (The Human Protein Atlas, http://www.proteinatlas.org/ ENSG00000174640SLCO2A1/ antibody). The antibody presumably detected a nontruncated SLCO2A1 protein, and, therefore, immunohistochemistry showed a false positive result, even though the protein was dysfunctional. Since truncated mutant alleles have been reported to account for approximately two-thirds of the mutated alleles found in patients with CEAS, it can be presumed that more than $60 \%$ of cases of CEAS can be correctly diagnosed with the use of immunohistochemical staining for SLCO2A1.

This study has several limitations. In the first place, the rarity of CEAS did not allow us to examine a large number of patients with the disease. Actually, three of four patients with CEAS were included in our previous study dealing with surgically removed intestine. ${ }^{11}$ However, we could show in the present study that biopsy specimens from apparently normal gastroduodenal mucosa, which can be easily obtained by endoscopy, may be valuable for differentiating CEAS from $\mathrm{CD}$. In the second place, the retrospective nature of our investigation did not allow for genetic analysis of SLCO2A1 in our CD patients. In fact, two out of our 603 patients with a diagnosis of $C D$ had a genetic diagnosis of CEAS in our prior investigation. ${ }^{6}$ Because genetic analysis is a gold standard for the distinction of CEAS from $\mathrm{CD}$, a comparison of genetic diagnosis and immunohistochemical features are inevitable to validate the results of our present investigation. However, we believe that immunohistochemical staining for SLCO2A1 expression together with confirmation of clinical characteristics, that is, chronic blood loss and protein 
loss through persistent, intractable small bowel ulcers, are essential to screen subjects who are candidates for genetic analysis of SLCO2A1.

In conclusion, immunohistochemical staining for SLCO2A1 in gastroduodenal tissues obtained by endoscopic biopsy may be useful for the distinction of CEAS from CD. With the use of the immunostaining, approximately two-thirds of patients with CEAS could be correctly diagnosed.

\section{CONFLICTS OF INTEREST}

T.M. has received grant support from AbbVie, Japan. The other authors declare that they have no conflicting interests.

\section{ACKNOWLEDGEMENTS}

This work was supported in part by a grant from the Japan Agency for Medical Research and Development (AMED) (15ek0109053h0002).

The authors would like to thank Professor Tadakazu Hisamatsu, Kyorin University, Mitaka, Japan for his valuable comments on the immunohistochemical study of the SLCO2A1 protein.

\section{REFERENCES}

1. Matsumoto T, Iida M, Matsui T, Yao T. Chronic nonspecific multiple ulcers of the small intestine: a proposal of the entity from Japanese gastroenterologists to Western enteroscopists. Gastrointest Endosc 2007;66(3 Suppl):S99-S107.

2. Matsumoto T, lida M, Matsui T, et al. Non-specific multiple ulcers of the small intestine unrelated to non-steroidal anti-inflammatory drugs. J Clin Pathol 2004;57:1145-1150.

3. Esaki M, Umeno J, Kitazono T, Matsumoto T. Clinicopathologic features of chronic nonspecific multiple ulcers of the small intestine. Clin J Gastroenterol 2015;8:57-62.

4. Matsumoto T, Nakamura S, Esaki M, et al. Endoscopic features of chronic nonspecific multiple ulcers of the small intestine: comparison with nonsteroidal anti-inflammatory drug-induced enteropathy. Dig Dis Sci 2006;51:1357-1363.

5. Matsumoto T, Kubokura N, Matsui T, Iida M, Yao T. Chronic nonspecific multiple ulcer of the small intestine segregates in offspring from consanguinity. J Crohns Colitis 2011;5:559-565.

6. Umeno J, Hisamatsu T, Esaki M, et al. A hereditary enteropathy caused by mutations in the SLCO2A1 gene, encoding a prostaglandin transporter. PLoS Genet 2015;11:e1005581.

7. Hosoe N, Ohmiya N, Hirai F, et al. Chronic enteropathy associated with SLCO2A1 gene [CEAS]-characterisation of an enteric disorder to be considered in the differential diagnosis of Crohn's disease. J Crohns Colitis 2017;11:1277-1281.
8. Uchida K, Nakajima A, Ushijima K, et al. Pediatric-onset chronic nonspecific multiple ulcers of small intestine: a nationwide survey and genetic study in Japan. J Pediatr Gastroenterol Nutr 2017;64:565-568.

9. Compton RF, Sandborn WJ, Yang H, et al. A new syndrome of Crohn's disease and pachydermoperiostosis in a family. Gastroenterology 1997;112:241-249.

10. Shim YW, Suh JS. Primary hypertrophic osteoarthropathy accompanied by Crohn's disease: a case report. Yonsei Med J 1997;38:319-322.

11. Yamaguchi S, Yanai S, Nakamura S, et al. Immunohistochemical differentiation between chronic enteropathy associated with SLC02A1 gene and other inflammatory bowel diseases. Intest Res 2018;16:393-399.

12. Hisabe T, Hirai F, Matsui T, Watanabe M. Evaluation of diagnostic criteria for Crohn's disease in Japan. J Gastroenterol 2014;49:9399.

13. Yamamoto H, Sekine Y, Sato Y, et al. Total enteroscopy with a nonsurgical steerable double-balloon method. Gastrointest Endosc 2001;53:216-220.

14. Iddan G, Meron G, Glukhovsky A, Swain P. Wireless capsule endoscopy. Nature 2000;405:417.

15. Perlemuter G, Guillevin L, Legman P, Weiss L, Couturier D, Chaussade S. Cryptogenetic multifocal ulcerous stenosing enteritis: an atypical type of vasculitis or a disease mimicking vasculitis. Gut 2001;48:333-338.

16. Chang DK, Kim JJ, Choi H, et al. Double balloon endoscopy in small intestinal Crohn's disease and other inflammatory diseases such as cryptogenic multifocal ulcerous stenosing enteritis (CMUSE). Gastrointest Endosc 2007;66(3 Suppl):S96-S98.

17. Zhang Z, Xia W, He J, et al. Exome sequencing identifies SLC02A1 mutations as a cause of primary hypertrophic osteoarthropathy. Am J Hum Genet 2012;90:125-132.

18. Sasaki T, Niizeki H, Shimizu A, et al. Identification of mutations in the prostaglandin transporter gene SLCO2A1 and its phenotypegenotype correlation in Japanese patients with pachydermoperiostosis. J Dermatol Sci 2012;68:36-44.

19. Guda K, Fink SP, Milne GL, et al. Inactivating mutation in the prostaglandin transporter gene, SLCO2A1, associated with familial digital clubbing, colon neoplasia, and NSAID resistance. Cancer Prev Res (Phila) 2014;7:805-812.

20. Diggle CP, Parry DA, Logan CV, et al. Prostaglandin transporter mutations cause pachydermoperiostosis with myelofibrosis. Hum Mutat 2012;33:1175-1181.

21. Busch J, Frank V, Bachmann N, et al. Mutations in the prostaglandin transporter SLCO2A1 cause primary hypertrophic osteoarthropathy with digital clubbing. J Invest Dermatol 2012;132:24732476. 